\title{
The symbiosis of contact force catheter use for hybrid ablation for atrial fibrillation
}

\author{
N. Kumar $\cdot$ L. Pison $\cdot$ P. Lozekoot $\cdot$ R. Choudhury $\cdot$ \\ M. La Meir $\cdot$ S. Gelsomino $\cdot$ H. Crijns $\cdot$ J. Maessen
}

Published online: 8 July 2015

(C) The Author(s) 2015. This article is published with open access at Springerlink.com

\begin{abstract}
Objective Reconduction across an ablation line is a common reason for arrhythmia recurrence over time. The hybrid procedure combines epicardial ablation of the pulmonary vein $(\mathrm{PV})$ and creation of a box lesion with endocardial touch-ups for any electrical gaps. A high contact force (CF) between the ablation tip and cardiac tissue may increase the risk of thrombus formation, catheter tip charring, steam pop formation, and even cardiac perforation. CF monitoring is a significant new parameter for titration of the $\mathrm{CF}$ for creating an adequate lesion.

Methods Thirty-eight consecutive patients underwent epicardial ablation using bipolar radiofrequency devices. After checking electrical bidirectional block of the ablation lines, an endocardial $\mathrm{CF}$ catheter was used for further ablation (if needed) to complete the isolation of PVs, box lesion, cavotricuspid isthmus (CTI), and complex fractionated atrial electrograms (CFAE).

Results Endocardial touch-up was needed for 2 PVs (1.3\%) and $10(26.3 \%)$ box lesions. It was also used for the CTI
\end{abstract}

\footnotetext{
N. Kumar $(\bowtie) \cdot$ L. Pison $\cdot$ H. Crijns

Department of Cardiology, Maastricht University Medical Centre and Cardiovascular Research Institute Maastricht (CARIM), P. Debyelaan 25,

PO Box 5800, 6229 HX Maastricht, The Netherlands

e-mail: drnarendra007kr@gmail.com

R. Choudhury

Department of Cardiology, AZ Sint- Jan Brugge- Oostende AV, Brugge, Belgium

P. Lozekoot · M. La Meir · S. Gelsomino · J. Maessen Department of Cardiac Surgery,

Maastricht University Medical Centre and Cardiovascular

Research Institute Maastricht (CARIM),

Maastricht, The Netherlands
}

line in $7(18.4 \%)$ patients, atrial tachycardia in $3(7.9 \%)$ patients, and additional CFAE ablation in 17 (44.7\%) patients. All 5 patients with arrhythmia recurrence had a mean $\mathrm{CF}<10 \mathrm{~g}(p=0.03)$. Procedure duration was significantly shorter in the CF group ( $223 \pm 57$ vs. $256 \pm 60 \mathrm{~min}, p=0.03$ ) compared with control group.

Conclusion Use of CF catheters is safe, feasible, and complementary to a hybrid procedure setup for atrial fibrillation ablation. Its real-time monitoring may predict future arrhythmia recurrence, and decrease procedure time.

Keywords Catheter ablation - Atrial fibrillation . Hybrid procedure $\cdot$ Contact force $\cdot$ Surgical ablation

\section{Introduction}

Atrial fibrillation (AF) is the most common arrhythmia globally affecting more than 20 million people and is a leading cause of stroke among people 65 years and older [1].

During catheter ablation for a cardiac arrhythmia, energy is delivered to areas of interest of the heart muscle that are causing the abnormal heart rhythm. Reconduction across an ablation line is one of the most frequent reasons for arrhythmia recurrence over time, e.g. pulmonary vein (PV), and linear lesions [2]. The hybrid procedure combines the epicardial ablation of PVs and creation of the box lesion by the cardiac surgeon and endocardial touch-ups (only if needed) to ensure ablation of any missed electrical gaps, by the electrophysiologist [3-5]. To create a transmural lesion, contact force (CF) between the ablation tip and cardiac tissue is a major determining parameter for radiofrequency catheters, thus its monitoring assumes significance [6]. Low CF may lead to an inadequate lesion. However, if it is very high, it may increase the risk of significant complications such as 
thrombus formation, catheter tip charring, steam pop formation and also cardiac perforation. To date CF catheters have never been used in a hybrid procedure setting for $\mathrm{AF}$ ablation.

To the best of our knowledge, this is the first article to study the safety and feasibility of using a CF catheter during the hybrid ablation of AF.

\section{Methods}

\section{Study population}

Thirty-eight patients with symptomatic AF underwent the hybrid procedure using the epicardial bipolar RF devices and an endocardial CF catheter. The definitions of paroxysmal, persistent, long-standing persistent, and permanent $\mathrm{AF}$, success and failure of ablation, and follow-up monitoring were based on the consensus statement of the Heart Rhythm Society, the European Heart Rhythm Association, and European Cardiac Arrhythmia Society.

Selection criteria of AF patients for this procedure were previously failed catheter ablation, failure of at least one antiarrhythmic drug (AAD), left atrial volume $\geq 55 \mathrm{ml}$, or patient preference for a hybrid procedure instead of a percutaneous approach. Prior catheter ablation was noted in the history for 18/38 AF patients (47.4\%) and 11/38 patients (29\%) had prior catheter ablation for cavo-tricuspid isthmus (CTI) dependent atrial flutter. The preoperative work-up consisted of transthoracic echocardiography, cardiac computed tomography (for assessing PV anatomy and integration to electroanatomical mapping), and pulmonary function testing.

\section{Control group}

A random, retrospective and randomised control group of 30 patients with similar patient characteristics (Table 1) was selected, to compare the endocardial ablation parameters, e.g. fluoroscopy time, procedure time and ablation time with a similar ablation setting of the radiofrequency generator. A standard non-force sensing irrigated tip catheter (ThermoCool, Biosense Webster) was used during a hybrid procedure for the patients in the control group.

\section{Hybrid procedure}

The hybrid procedure was performed as described elsewhere $[3,4,7]$. Briefly, all AADs were stopped before the procedure (considering their half-life) except amiodarone. Under general anaesthesia, after introducing two $5 \mathrm{~mm}$ working ports and a $5 \mathrm{~mm}$ video port in the intercostal spaces of the left and right hemithorax, patients underwent $\mathrm{PV}$ isolation using epicardial bipolar radiofrequency clamps (Atricure,
Table 1 Patient characteristics

\begin{tabular}{ll}
\hline Variables & Number \\
\hline Patients $(n)$ & 38 \\
Gender (men/women) & $34 / 4$ \\
Age (years) & $61.8 \pm 7.2$ \\
BMI $\left(\mathrm{kg} / \mathrm{m}^{2}\right)$ & $28.2 \pm 3.4$ \\
Tested pulmonary veins $(n, \%)$ & $150(100)$ \\
Touch up needed in pulmonary veins $(n, \%)$ & $2(1.3)$ \\
Tested box lesions $(n, \%)$ & $37(97.4)$ \\
Touch up needed in box $(n, \%)$ & $10(27)$ \\
Additional CTI ablation $(n, \%)$ & $7(18.4)$ \\
Additional atrial tachycardia ablation $(n, \%)$ & $3(7.9)$ \\
Additional mitral line ablation $(n, \%)$ & $0(0)$ \\
Additional CFAE ablation $(n, \%)$ & $17(44.7)$ \\
Average procedure time (minutes) & $223 \pm 57$ \\
Medical history & \\
Hypertension $(n, \%)$ & $16(42.1)$ \\
Diabetes $(n, \%)$ & $5(13.2)$ \\
CAD $(n, \%)$ & $15(39.5)$ \\
COPD $(n, \%)$ & $2(5.3)$ \\
Medication use & \\
Amiodarone $(n, \%)$ & $5(13.2)$ \\
Flecainide $(n, \%)$ & $19(50)$ \\
Sotalol $(n, \%)$ & $8(21.0)$ \\
Disease characteristics &
\end{tabular}

\section{Disease characteristics}

Paroxysmal/persistent/permanent AF $(n / n / n$, $\% / \% / \%)$

$\mathrm{AF}$ duration (years, mean $\pm \mathrm{SD}$ )

$14,19,5(37,50,13)$

$\operatorname{LVEF}(\%$, mean \pm SD)

$6.8 \pm 4.352 \pm 14$

$93 \pm 25$

$18(47.4)$

of prior catheter ablations $(n, \%)$

Number of prior electrical/pharmacological $26(68.4)$

cardioversions $(n, \%)$

$\overline{A F}$ atrial fibrillation, $A F l$ atrial flutter, $B M I$ body mass index, $n$ numbers, $C T I$ cavo tricuspid isthmus, $C A D$ coronary artery disease, $C F A E$ complex fractionated atrial electrograms, $C H F$ congestive heart failure, $L V E F$ left ventricle ejection fraction, $L A$ left atrial, $S D$ standard deviation

West Chester, OH, USA). An epicardial box lesion was made using a bipolar radiofrequency pen or a linear pen device (Coolrail, Atricure) consisting of a roof line (connecting both superior PVs) and an inferior line (connecting both inferior PVs). Using left femoral vein access, catheters were placed in the His bundle and coronary sinus. Using a single transseptal puncture a circular mapping catheter, according to the size of the PV (Lasso, Biosense Webster Inc., Diamond Bar, USA), was placed ostially to assess the PV potentials. The electrophysiologist checked for electrical isolation of ablation lines by pacing manoeuvres, electroanatomical mapping and/or adenosine bolus administration. A CARTO platform (CARTO-3; Biosense Webster, Diamond Bar, CA) was used for electroanatomical mapping. A left atrium shell was created using point-by-point acquisition with the aid of the $\mathrm{CF}$ catheter (SmartTouch ${ }^{\mathrm{TM}}$, Biosense Webster Inc.). Criteria for an adequate left atrial shell 
Fig. 1 Endocardial radiofrequency ablation (red dots) using $\mathrm{CF}$ catheter for completion of the inferior line of box lesion. a At start of ablation. b After finishing ablation. We can further notice the visual change in the voltage map after finishing the ablation
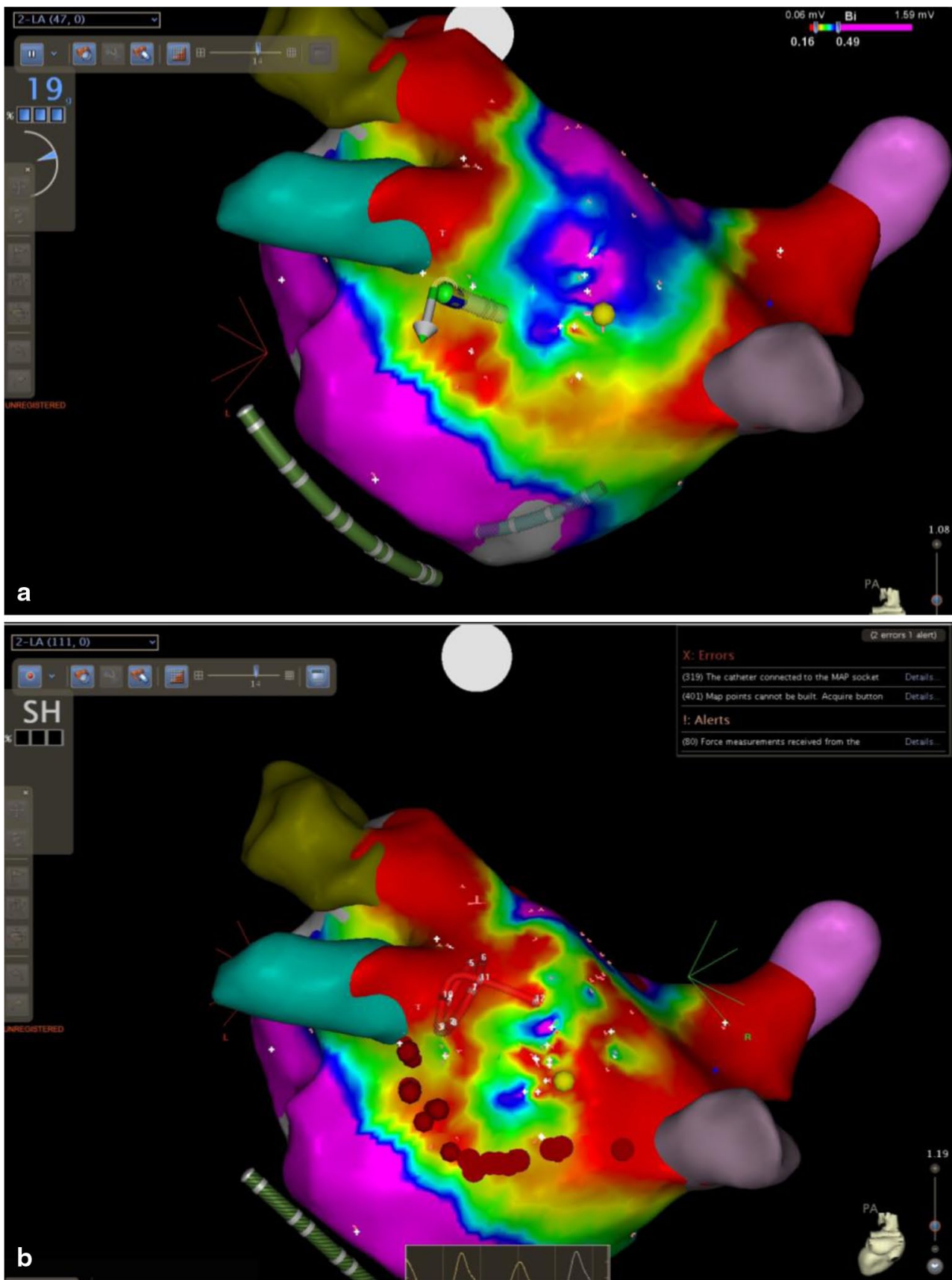

were: $\geq 100$ points, which were homogenously distributed to create the entire chamber.

The CF catheter was further used for endocardial ablation (if needed) to complete the PV isolation and box lesion, while maintaining the CF values between 10-40 G with the aid of a Stockert 70 radiofrequency generator (Biosense Webster) with a maximal temperature of $43^{\circ} \mathrm{C}$ and limited to $25 \mathrm{~W}$ at the posterior wall (Fig. 1). Endocardial ablation was also used for CTI ablation in patients with a prior history of atrial flutter, any inducible atrial tachycardia, and complex fractionated atrial electrogram (CFAE) ablation is used in case of persistent AF after epicardial PV isola- tion and box lesion. The spatial distribution of CFAE in the left atria of AF patients was evaluated using software designed to automatically identify CFAE in the framework of the CARTO platform. In detail, the minimal amplitude threshold and maximal amplitude threshold were set to \pm 0.05 millivolt $(\mathrm{mV})$ and $\pm 0.15 \mathrm{mV}$, and the minimal and maximal intervals between two consecutive peaks were set to $60 \mathrm{msec}$ and $120 \mathrm{msec}$ [8].

After finishing the endocardial ablation, and retesting for arrhythmia, the induction was performed. The left atrial appendage (LAA) was removed using a stapling device in 11 patients. Finally, bilateral chest drains were inserted in 
the pleural cavities. Patients were extubated in the operating room before being transferred to the ICU. Within $6 \mathrm{~h}$, lowmolecular-weight heparin was started and acenocoumarol was restarted after 2 days. AADs were restarted as soon as possible. After confirming absence of atrial arrhythmias after 6 months, AADs and anticoagulation were discontinued.

Follow-up

Follow-up was conducted at our hospital's outpatient clinic in the 3rd, 6th, 9th, 12th month after the procedure. A 7-day Holter monitoring was used for all patients; if not available patients underwent at least 48-hour Holter monitoring (3 patients). Arrhythmia recurrence was considered to be any episode of AF, flutter or tachycardia lasting more than $30 \mathrm{~s}$ detected after the 3-month blanking period.

\section{Statistical analysis}

Continuous variables are expressed as mean \pm standard deviation. Data were retrospectively entered into a database. Statistical analysis was performed using SPSS 16.0 (SPSS Inc, Chicago, IL). Event-free survival was estimated using Kaplan-Meier and compared by the log-rank test. Possible predictors of arrhythmia recurrence were performed using the Cox proportional hazards regression models. A $p$ value of $<0.05$ was considered significant.

\section{Results}

The study group consisted of 38 patients $(61.8 \pm 7.2$ years; 34 male) with AF duration of $6.8 \pm 4.3$ years. A left-sided common ostium was observed in 2 patients. The body mass index was $28.2 \pm 3.4 \mathrm{~kg} / \mathrm{m}^{2}$. Eighteen patients had undergone catheter PV isolation. Electrical/pharmacological cardioversions were attempted previously for 26 patients $(68.4 \%)$. The mean procedure time (from initial skin incision to skin closure) was $223 \pm 57 \mathrm{~min}$. The mean left atrium size was $93 \pm 25 \mathrm{ml}$, with a mean left ventricular ejection fraction of $52 \pm 14 \%$. Epicardial bipolar radiofrequency devices were used for 150 PVs $(100 \%)$ and 37 box lesions $(100 \%)$ for 38 patients. Endocardial touch-up using a CF catheter was needed for 2 PVs $(1.3 \%)$ of different patients, i.e. left superior PV and right inferior PV, and for 10 box lesions $(26.3 \%)$. A total of 4 touch-up lesions with a mean duration of $39 \mathrm{~s}$ were applied to the PVs. It was also used for creation of a CTI line in $7(18.4 \%)$ patients and additional CFAE ablation in 17 (44.7\%) patients. Further, during burst pacing to test for inducible AF, 3 (7.9\%) patients developed 5 different morphologies of inducible atrial tachycardia and henceforth CF was also used for endocardial ablation for all of them. The mean grams of force per ablation lesion were
$17.6 \pm 7.8 \mathrm{~g}$ for the right PVs and 15.7 $\pm 7.2 \mathrm{~g}$ for the left PVs. Further details on the lesion sets and patient characteristics are shown in Table 1.

The control group consisted of 30 patients (paroxysmal: $11(37 \%)$, persistent: $16(53 \%)$, permanent $=3(10 \%))$. The mean left atrial volume was $91 \pm 23 \mathrm{ml}$. The statistical analysis (using the Mann-Whitney test) for procedure duration was significantly shorter in the $\mathrm{CF}$ group $(223 \pm 57$ vs. $256 \pm 60 \mathrm{~min}, p=0.03$ ) compared with the control group. There were no statistically significant differences observed in ablation time $(11.2 \pm 5.9$ vs $14.1 \pm 6.2 \mathrm{~min})$ or fluoroscopy time ( $18.5 \pm 8.6$ vs $23.4 \pm 6.4 \mathrm{~min})$ although all were reduced in the CF group. No difference in complications was seen between the groups. After a mean follow-up of $357 \pm 43$ days (395-334 days) for control group patients, $75 \%$ of patients were AF free without AADs.

\section{Follow-up}

During the first 3 months after the procedure, the patients were blanked for the analysis of recurrence of AF. After follow-up of $335 \pm 66.5$ days (410-320 days), 33/38 patients $(86.8 \%)$ of the study population were in sinus rhythm without AADs, 2 patients needed AADs to maintain sinus rhythm. Catheter ablation was needed for 3 patients i.e. 1 typical CTI dependent atrial flutter, 1 mitral isthmus dependent flutter, and an atrial tachycardia. Non-inducible patients, who were tested immediately after ablations, had less arrhythmia during follow-up than inducible ones $(p=0.04)$. Among all patients with recurring arrhythmia, we retrospectively observed that they had received endocardial touch-up; the mean CF was $<10 \mathrm{~g}(p=0.03)$, in spite of continuous attempts to improve the contact. Figure 3 shows a Kaplan-Meier graph of AF-free survival.

\section{Adverse events}

No thromboembolic complications or conversion to cardiopulmonary bypass were encountered. No signs of infection and phrenic nerve paralysis were seen among any of the patients. One patient had recurrent pain in the left thorax region a few weeks after the procedure. Diagnosis of atraumatic lung hernia was confirmed by high-resolution computerised tomographic (HRCT) $[9,10]$. Retrospectively, the aetiology was attributed to widening of the mini invasions of $12 \mathrm{mms}$ (initially) to $3 \mathrm{~cm}$ to accommodate an epicardial left atrial appendage (LAA) clip. To the best of our knowledge, this is the only case of atraumatic lung hernia secondary to usage of an LAA clip in the literature. No other complications were associated with such clip usage. No signs of PV stenosis were seen in any patient during follow-up HRCT [11]. 
Fig. 2 Temporal display of change of contact force (grey colored) with time during endocardial radiofrequency ablation (red dots)

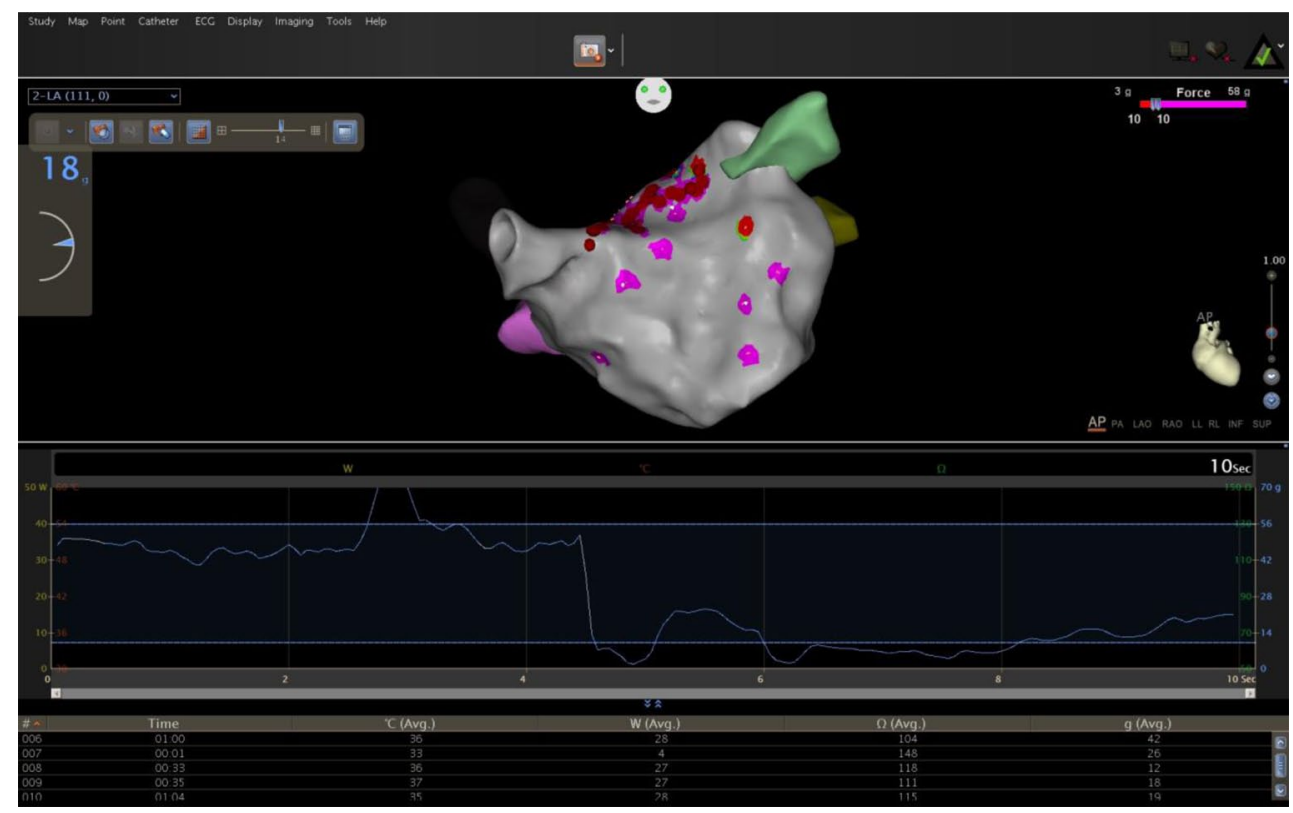

\section{Discussion}

This manuscript describes, for the first time, combined simultaneous thoracoscopic epicardial ablation using bipolar devices and endocardial ablation using CF catheters for paroxysmal and persistent AF. Further, the use of a CF catheter results in superior transmurality of linear ablation lesions.

The pharmacological approach with AADs has not achieved optimal results in the long-term maintenance of sinus rhythm in patients with AF [12-14]. Considering the limitations of medical therapy, there has been an upsurge in catheter and surgical ablation techniques for AF ablation [15-17]. The recently proposed hybrid approach combines the advantages of individual approaches while simultaneously doing away with their disadvantages, complications, and ultimately ensuring the transmurality of lesions $[3,5$, 18]. AF recurrence is often associated with PV reconnection after initial PV ablation [19-23]. It is attributed to the inability of endocardial catheter based ablation techniques to guarantee long-lasting transmurality of the lesions [2].

Both ablation technologies used in this setup share common principles. An epicardial bipolar radiofrequency device (Atricure Inc.) works on the principle of impedance change and endocardial ablation using a $\mathrm{CF}$ catheter works on $\mathrm{CF}$ measurement. Interestingly, several studies have correlated impedance drop with $\mathrm{CF}$ with due credit to the more detailed quantitative analysis and use of nonparametric statistics [24-26]. Martinek et al. found that the use of CF sensing technology catheters significantly reduces ablation time, translating to an overall reduction in procedural times in PV isolation. This reasoning is well suited to the hybrid procedure as well. Avoiding

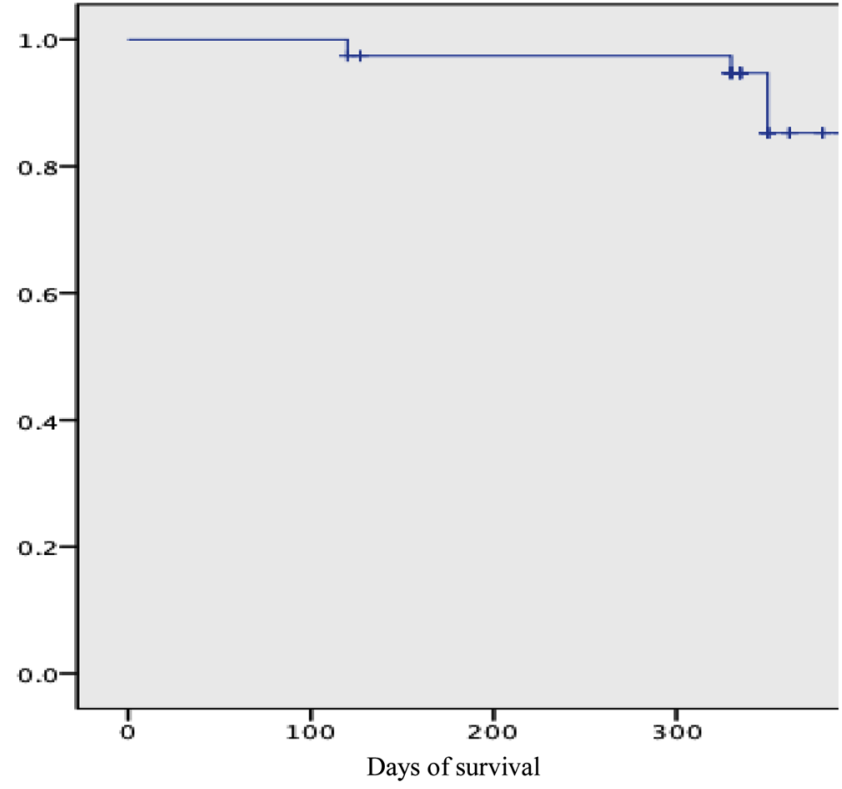

Fig. 3 Kaplan-Meier survival curve of atrial arrythmia free survival after a single hybrid procedure after a blanking period of 90 days for the arrhythmias. (DAYS $=$ follow up in days)

radiofrequency delivery in areas with insufficient tissue electrode contact substantially reduced energy delivery. These catheters also significantly reduced ablation and procedural times in PV isolation [6]. Continuous monitoring of $\mathrm{CF}$ values may help to provide extra helpful information to the operating physician for safer catheter manipulation. It helps to make an $\mathrm{AF}$ ablation procedure safer as high $\mathrm{CF}$ values may occur, not only during catheter manipulation but also during ablation (Fig. 2 and video 1, [27]). 
Higher incidence of cardiac tamponade is seen with endocardial left atrial linear ablations [28]. When endocardial tissue is exposed to high levels of radiofrequency energy, it may lead to cardiac tamponade with steam pop [29]. Other than radiofrequency energy, CF is a significant cofactor for cardiac rupture and thrombus. Several preclinical studies have associated it with significant increases in radiofrequency lesion depth, diameter, and volume [30-33].

Non-inducible patients tested immediately after ablations (using adenosine bolus, burst pacing and electrical testing) had less arrhythmias during follow-up than inducible ones [34]. This further signifies the importance of testing protocols used at the end of procedures. Lesion size is significantly affected by CF, e.g. lower radiofrequency power $(30 \mathrm{~W})$ and high CF $(30-40 \mathrm{~g})$ produces larger and deeper lesions while compared with the ones produced at greater power $(50 \mathrm{~W})$ but lower CF. Different anatomical sites in the left atrium can be difficult to approach with a catheter. Thus continuous measurement of catheter-tissue CF during radiofrequency ablation assumes significance to achieve good quality lesions [35].

There was significantly less need for endocardial touchups for epicardial ablation of PVs compared with other epicardial ablations, which again reminds us of the robustness of epicardial clamps compared with the epicardial pen used for box lesions. Procedural duration and ablation efficacy are significantly determined by catheter-tissue CF. Moreover, the number of lesions with low average $\mathrm{CF}$ or force time integral (FTI) and the time to achieve acute PV isolation are linearly related [36, 37]. Acute PV reconnection has been strongly associated with low values of CF and FTI [33]. In our study too, reconduction zones were localised in areas with mean $\mathrm{CF}$ readings $<10 \mathrm{~g}$, in spite of continuous attempts to improve the contact. Low CF can predict gap formation in ablation FTI $[35,37]$ Moreover, Kumar et al. reported that optimal CF might be easier to obtain and maintain during right as compared with left PV ablation [35]. In our study too, the time with poor CF values $(5 \mathrm{~g})$ was significantly shorter for right than for left PV ablation [38].

The LAA was not excluded among the remaining patients due to anatomical restrictions or surgeon preference. LAA exclusion may eliminate the extra PV triggers of $\mathrm{AF}$, and might also further decrease stroke incidence in patients with a high CHA2DS2-VASc score [39, 40] but can also cause bleeding [41], which might require conversion to sternotomy. Its effect on left atrial function and remaining thromboembolic risk is incompletely understood [42]. LAA removal has not always been associated with a higher success rate [43]. So, in future as more patients are receiving such LAA exclusion therapies, its role to determine the success of an AF ablation will be worth looking for.

The strength of this study lies in its exclusive approach to AF ablation and good follow-up. Further, this procedure is less invasive with minimum complications, unlike the pure surgical approach. The wastage of unnecessary energy delivery with CF catheters was avoided in three way: firstly by simultaneous epicardial ablation, secondly by visualising the electrical gaps by pacing manoeuvres and electroanatomical mapping and, finally by avoiding ablations in locations with insufficient surface contact. Moreover, the success rate of this procedure is comparable with either approach individually, especially considering the dilated left atrium and long years of AF duration. Monitoring of $\mathrm{CF}$ values during ablation can control the success rate of the procedure.

\section{Study limitations}

The small number of patients and a control group in this single-centre retrospective study prevents definitive conclusions. However, future larger, long-term and multicentre studies may corroborate our results.

\section{Conclusion}

Use of CF catheters is safe, feasible, and complementary to a hybrid procedure setup for AF ablation. The monitoring of real-time $\mathrm{CF}$ values predicts future arrhythmia recurrence and decreases procedure time.

Acknowledgement The authors thank Niky Claessens, RN; Ruud Duijsings, RN, and Luuk Debie, RN, for the technical support.

Disclosure Mark La Meir and Laurent Pison are consultants to Atricure. The other authors have no conflict of interests to declare.

Open Access This article is distributed under the terms of the Creative Commons Attribution Noncommercial License which permits any noncommercial use, distribution, and reproduction in any medium, provided the original author(s) and source are credited.

\section{References}

1. Kannel WB, Abbott RD, Savage DD, McNamara PM. Epidemiologic features of chronic atrial fibrillation: the Framingham study. N Engl J Med. 1982;306(17):1018-22.

2. Medi C, Sparks PB, Morton JB, et al. Pulmonary vein antral isolation for paroxysmal atrial fibrillation: results from long-term follow-up. J Cardiovasc Electrophysiol. 2011;22(2):137-41.

3. La Meir M, Gelsomino S, Luca F, et al. Minimally invasive thoracoscopic hybrid treatment of lone atrial fibrillation: early results of monopolar versus bipolar radiofrequency source. Interact Cardiovasc Thorac Surg. 2012;14(4):445-50.

4. Kumar N, Pison L, La Meir M, Maessen J, Crijns HJ. Hybrid approach to atrial fibrillation ablation using bipolar radiofrequency devices epicardially and cryoballoon endocardially. Interact Cardiovasc Thorac Surg. 2014;19(4):590-4. 
5. Kumar N, Bonizzi P, Pison L et al. Impact of hybrid procedure on $\mathrm{P}$ wave duration for atrial fibrillation ablation. J Interv Card Electrophysiol. 2015. doi:10.1007/s10840-014-9969-9.

6. Martinek M, Lemes C, Sigmund E, et al. Clinical impact of an open-irrigated radiofrequency catheter with direct force measurement on atrial fibrillation ablation. Pacing Clin Electrophysiol. 2012;35(11):1312-8.

7. Kumar N, Pison L, la Meir M, Maessen J. Direct visualization of pulmonary vein stenosis after previous catheter ablation. Heart Rhythm. 2014. doi:10.1016/j.hrthm.2013.10.052.

8. Wu J, Estner H, Luik A, et al. Automatic 3D mapping of complex fractionated atrial electrograms (CFAE) in patients with paroxysmal and persistent atrial fibrillation. J Cardiovasc Electrophysiol. 2008;19(9):897-903.

9. Kumar N, Pison L, La Meir M, Maessen J. Atraumatic lung hernia: a rare complication of minimally invasive surgical atrial fibrillation ablation. J Atrial Fib. 2013;6(4):3.

10. Kumar N, Timmermans C, Das M, Pison L, Maessen J, Crijns H. Re: CT imaging of complications of catheter ablation for atrial fibrillation. Clin Radiol. 2014;69(8):e367-8.

11. Kumar N, Phan K, Aksoy I, et al. Management of pulmonary vein stenosis following catheter ablation of atrial fibrillation. J Atrial Fib. 2014;7(1):7.

12. Chimienti M, Cullen MT Jr, Casadei G. Safety of long-term flecainide and propafenone in the management of patients with symptomatic paroxysmal atrial fibrillation: report from the Flecainide and Propafenone Italian Study Investigators. Am J Cardiol. 1996;77(3):60-75A.

13. Wyse DG, Waldo AL, DiMarco JP, et al. A comparison of rate control and rhythm control in patients with atrial fibrillation. N Engl $\mathrm{J}$ Med. 2002;347(23):1825-33.

14. Jost N, Kohajda Z, Kristof A, et al. Atrial remodeling and novel pharmacological strategies for antiarrhythmic therapy in atrial fibrillation. Curr Med Chem. 2011;18(24):3675-94.

15. Haissaguerre M, Gencel L, Fischer B, et al. Successful catheter ablation of atrial fibrillation. J Cardiovasc Electrophysiol. 1994;5(12):1045-52.

16. Cox JL. The first Maze procedure. J Thorac Cardiovasc Surg. 2011;141(5):1093-7.

17. Vries LJ de, Akca F, Khan M et al. Clinical outcome of ablation for long-standing persistent atrial fibrillation with or without defragmentation. Neth Heart J. 2014;22(1):30-6.

18. Kumar N, Pison L, Blaauw Y, et al. Pulmonary vein stenosis after laser balloon ablation for atrial fibrillation. JACC: Clin Electrophysiol. 2015. doi:10.1016/j.jacep.2015.04.001

19. Ouyang F, Bansch D, Ernst S, et al. Complete isolation of left atrium surrounding the pulmonary veins: new insights from the double-Lasso technique in paroxysmal atrial fibrillation. Circulation. 2004;110(15):2090-6.

20. Verma A, Kilicaslan F, Pisano E, et al. Response of atrial fibrillation to pulmonary vein antrum isolation is directly related to resumption and delay of pulmonary vein conduction. Circulation. 2005;112(5):627-35.

21. Kumar N, Bonizzi P, Lankveld T, Rad MM. Left atrial dyssynchrony time measured by tissue Doppler imaging to predict atrial fibrillation recurrences after pulmonary vein isolation: is this a mirage or the panacea? Anatol J Cardiol. 2015;15(2):123-4.

22. Yaksh A, Kik C, Knops P, et al. Atrial fibrillation: to map or not to map? Neth Heart J. 2014;22(6):259-66.

23. Kumar N, Dinh T, Magdi Abbas M et al. Failure to reach the optimal temperature during cryoablation due to refrigerant cylinder problem. Res Cardiovasc Med. 2015;4(1):e25592.

24. Ullah W, Hunter RJ, Baker V, et al. Target indices for clinical ablation in atrial fibrillation: insights from contact force, electrogram, and biophysical parameter analysis. Circ Arrhythm Electrophysiol. 2014;7(1):63-8.
25. Nakagawa H, Kautzner J, Natale A, et al. Locations of high contact force during left atrial mapping in atrial fibrillation patients: electrogram amplitude and impedance are poor predictors of electrode-tissue contact force for ablation of atrial fibrillation. Circ Arrhythm Electrophysiol. 2013;6(4):746-53.

26. Kumar S, Haqqani HM, Chan M, et al. Predictive value of impedance changes for real-time contact force measurements during catheter ablation of atrial arrhythmias in humans. Heart Rhythm. 2013;10(7):962-9.

27. Kuck KH, Reddy VY, Schmidt B, et al. A novel radiofrequency ablation catheter using contact force sensing: toccata study. Heart Rhythm 2012;9(1):18-23.

28. Jais P, Shah DC, Haissaguerre M, et al. Efficacy and safety of septal and left-atrial linear ablation for atrial fibrillation. Am J Cardiol 1999;84(9A):139-46R.

29. Hsu LF, Jais P, Hocini M, et al. Incidence and prevention of cardiac tamponade complicating ablation for atrial fibrillation. Pacing Clin Electrophysiol 2005;28(Suppl 1):S106-9.

30. Wittkampf FH, Nakagawa H. RF catheter ablation: lessons on lesions. Pacing Clin Electrophysiol. 2006;29(11):1285-97.

31. Yokoyama K, Nakagawa H, Wittkampf FH, Pitha JV, Lazzara R, Jackman WM. Comparison of electrode cooling between internal and open irrigation in radiofrequency ablation lesion depth and incidence of thrombus and steam pop. Circulation. 2006;113(1):11-9.

32. Nakagawa H, Yamanashi WS, Pitha JV, et al. Comparison of in vivo tissue temperature profile and lesion geometry for radiofrequency ablation with a saline-irrigated electrode versus temperature control in a canine thigh muscle preparation. Circulation. 1995;91(8):2264-73.

33. Eick OJ, Bierbaum D. Tissue temperature-controlled radiofrequency ablation. Pacing Clin Electrophysiol. 2003;26(3):725-30.

34. Kumar N, Pison L, La Meir M, Maessen J, Crijns HJ. Testing of box lesion by adenosine. J Atrial Fibrillation. 2013-2014;6(4):95.

35. Kumar S, Morton JB, Lee J, et al. Prospective characterization of catheter-tissue contact force at different anatomic sites during antral pulmonary vein isolation. Circ Arrhythm Electrophysiol. 2012;5(6):1124-9.

36. Thiagalingam A, D'Avila A, Foley L, et al. Importance of catheter contact force during irrigated radiofrequency ablation: evaluation in a porcine ex vivo model using a force-sensing catheter. J Cardiovasc Electrophysiol. 2010;21(7):806-11.

37. Neuzil P, Reddy VY, Kautzner J et al. Electrical reconnection after pulmonary vein isolation is contingent on contact force during initial treatment: results from the EFFICAS I study. Circ Arrhythm Electrophysiol. 2013;6(2):327-33.

38. Stabile G, Solimene F, Calo L, et al. Catheter-tissue contact force for pulmonary veins isolation: a pilot multicentre study on effect on procedure and fluoroscopy time. Europace. 2014;16(3):335-40.

39. Lip GY, Nieuwlaat R, Pisters R, Lane DA, Crijns HJ. Refining clinical risk stratification for predicting stroke and thromboembolism in atrial fibrillation using a novel risk factor-based approach: the euro heart survey on atrial fibrillation. Chest. 2010;137(2):263-72.

40. Ohtsuka T, Ninomiya M, Nonaka T, Hisagi M, Ota T, Mizutani T. Thoracoscopic stand-alone left atrial appendectomy for thromboembolism prevention in nonvalvular atrial fibrillation. J Am Coll Cardiol. 2013;62(2):103-7.

41. Driessen AH, Krul SP, Mol BA de, Groot JR de. Second chance for a totally thoracoscopic video-assisted pulmonary vein isolation for atrial fibrillation. Ann Thorac Surg. 2012;93(6):2051-3.

42. Dawson AG, Asopa S, Dunning J. Should patients undergoing cardiac surgery with atrial fibrillation have left atrial appendage exclusion? Interact Cardiovasc Thorac Surg. 2010;10(2):306-11.

43. Krul SP, Pison L, La Meir M, et al. Epicardial and endocardial electrophysiological guided thoracoscopic surgery for atrial fibrillation: a multidisciplinary approach of atrial fibrillation ablation in challenging patients. Int J Cardiol. 2014;173(2):229-35. 


\title{
Advertisement placed here.
}

\author{
CSS Bohn

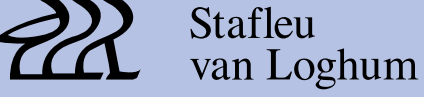 \\ Springer Media
}

Houten 2015 


\title{
Advertisement placed here.
}

\author{
CSS Bohn

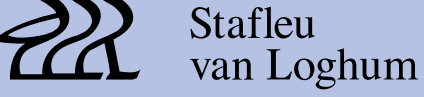 \\ Springer Media
}

Houten 2015 